\title{
Mineração
}

\section{Dimensionamento de espessadores: validação experimental de três técnicas convencionais}

\section{(Dimensioning of thickeners: experimental validation of three conventional techniques)}

\begin{abstract}
Thiago César de Souza Pinto
Doutorando em Engenharia Mineral, Departamento de Engenharia de Minas e de Petróleo, Escola Politécnica da USP, São Paulo-SP - Grupo de Pesquisa "Tratamento de Minérios". E-mail: thiago.cesar@usp.br

José Renato Baptista de Lima

Professor Associado, Departamento de Engenharia de Minas e de Petróleo, Escola Politécnica da USP São Paulo-SP - Grupo de Pesquisa "Tratamento de Minérios". E-mail: jrblima@usp.br

Deovaldo de Moraes Júnior

Professor Titular, Faculdade de Engenharia Química da Universidade Santa Cecília-Santos-SP

E-mail:deovaldo@unisanta.br

Laurindo de Salles Leal Filho

Professor Titular, Departamento de Engenharia de Minas e de Petróleo, Escola Politécnica da USP São Paulo-SP - Grupo de Pesquisa “Tratamento de Minérios". E-mail: lauleal@usp.br
\end{abstract}

\section{Resumo}

Esse trabalho faz revisão dos princípios utilizados por três importantes técnicas utilizadas para dimensionar espessadores: Fluxo de Sólidos, Talmadge-Fitch e Roberts. Elas foram aplicadas para validar o cálculo do diâmetro $(D)$ e a área de sedimentação $(A)$ de um espessador que clarifica salmoura em uma fábrica localizada em Cubatão-SP. O dimensionamento do espessador (cálculo de $A$ e $D$ ) foi baseado no comportamento da sedimentação, avaliado em escala de laboratório, de partículas de sal suspensas em salmoura. Os valores de $D$ e $A$ ( $\left.D=13,9-14,1 \mathrm{~m} ; A=151,7-156,1 \mathrm{~m}^{2}\right)$ obtidos pelas três técnicas foram comparados com o diâmetro do espessador real $(D=20,0 \mathrm{~m})$. Por outro lado, os valores corrigidos para $D$ e $A\left(D=18,1-18,3 \mathrm{~m} ; A=257,0-263,0 \mathrm{~m}^{2}\right)$ foram obtidos usando um fator de escala $(f=1,30)$. Esses valores são $10 \%$ menores em relação ao equipamento industrial.

Palavras-chave: Espessador, dimensionamento, fator de escala.

\begin{abstract}
This work reviews the fundamentals of three important techniques currently used to dimension thickeners: Flux of Solids, Talmadge-Fitch, and Roberts. They were applied to validate the calculation of diameter (D) and sedimentation area (A) of a real thickener, which clarifies brine in a plant located at Cubatão-SP. The calculation of thickener dimension ( $A$ and D) was based on the sedimentation behavior of salt particles in brine on a laboratory scale. The values of $D$ and $A\left(D=13,9-14,1 \mathrm{~m} ; A=151,7-156,1 \mathrm{~m}^{2}\right)$, which were calculated by the three techniques, were compared with the diameter of the real equipment $(D=20,0 m)$. On the other hand, corrected values of $D$ and $A(D=18,1-18,3 \mathrm{~m}$; $\left.A=257,0-263,0 \mathrm{~m}^{2}\right)$ were obtained by using a scale factor $(f=1,3)$. These results are $10 \%$ lower than the real equipment.
\end{abstract}

Keywords: Thickener, dimensioning, scale factor. 


\section{Introdução}

Espessadores são tanques de sedimentação empregados em um tipo particular de separação sólido-líquido, separação esta que é denominada de espessamento. Tais espessadores fazem uso da diferença de massa específica entre a fase líquida e as partículas sólidas que estão suspensas na referida fase líquida. No âmbito da tecnologia mineral, o espessamento é utilizado para aumentar a concentração de sólidos de polpas até valores convenientes para operações subseqüentes, como bombeamento, filtragem ou condicionamento com reagentes de flotação. Deste modo, a função dos espessadores é a de receber uma polpa diluída e gerar um produto (underflow) que exibe maior concentração de sólidos que a alimentação. Um segundo produto, (overflow), exibe concentração de sólidos menor que aquela apresentada pela alimentação. Via de regra, tal produto constitui a fase líquida clarificada.

O diâmetro de espessadores industriais é dimensionado através de determinadas técnicas, as quais são baseadas no estudo do comportamento da sedimentação de partículas sólidas no meio aquoso. Tal estudo é executado em bateladas, embora a unidade industrial opere em escala contínua. Essas técnicas, apesar de seu largo uso, apresentam deficiências em função das muitas variáveis do processo de espessamento e, também, da complexidade dos mecanismos de sedimentação de partículas sólidas em meio fluido. O presente trabalho teve por objetivo aplicar três metodologias convencionais (Fluxo de Sólidos ou Metcalf-Eddy, Talmadge-Fitch e Roberts) para validar o dimensionamento de um espessador industrial, que já se encontra em operação, comparando o diâmetro calculado pelas técnicas convencionais com o diâmetro real do equipamento industrial. Tal comparação se justifica, não somente como validação das técnicas tradicionais de dimensionamento, como também para discussão sobre fatores de escalonamento utilizados em projetos de espessadores industriais.

\section{Background}

As principais informações necessárias ao dimensionamento de espessadores são: capacidade requerida para alimentação, overflow e/ou underflow; características dos sólidos (tamanho, forma e massa específica das partículas); características do líquido (viscosidade e massa específica); estado de agregação/ dispersão das partículas suspensas no líquido e razão mássica sólido-líquido na suspensão que será submetida à operação unitária de espessamento (Metcalf et al, 1979).

Os fundamentos que regem o dimensionamento de espessadores começaram a ser estudados por Coe e Clevenger (1917) através de sedimentação em provetas. Eles propuseram colocar a polpa que se deseja espessar em provetas graduadas, monitorando-se em função do tempo $(\theta)$, a altura $(Z)$ da linha divisória (LD) entre o líquido já clarificado e o restante da polpa que estava sendo espessada. A representação gráfica do comportamento de $\mathrm{Z}$ versus $\theta$ foi denominada curva de sedimentação.

Talmadge et al. (1955), Roberts (1949) e Metcalf et al. (1979) utilizaram a curva de sedimentação para dimensionar, através de modelagem gráfica ou matemática, o diâmetro do tanque espessador e, como decorrência, a área necessária para se obter o desempenho desejado. $\mathrm{O}$ ferramental matemático inerente a cada método é sumarizado na Tabela 1 .

Metcalfe Eddy (1979) propõem que o dimensionamento do diâmetro de espessadores seja efetuado através de um método baseado no fluxo de sólidos, isto é, seja efetuado através da vazão mássica que passa por uma unidade de área durante certo intervalo de tempo, após o sistema atingir o estado estacionário. A curva do fluxo de sólidos em, função da concentração (Figura 1), sendo tangenciada por uma reta que parte do ponto onde se encontra a concentração de sólidos desejada no underflow $\mathrm{C}_{\mathrm{E}}$ (eixo das abscissas), indica o fluxo de sólidos limitante da operação $\left(\mathrm{F}_{\mathrm{L}}\right)$, quando tal fluxo toca o eixo das ordenadas.
Roberts (1949), analisando o trabalho de Coe e Clevenger, propôs um método gráfico que parte da curva de sedimentação ( $Z$ versus $\theta)$ traçada em escala monologarítmica. Esse método evidencia e caracteriza o ponto crítico (início da zona de compressão) com a descontinuidade da curva. O método, também, é realizado com ensaios em provetas com uma única concentração de alimentação (Figura 2), tendo, também, sido reportado por Gomide (1980).

Talmadge e Fitch (1955), propõem um método gráfico para o dimensionamento de espessadores. Tal método é subjetivo, pois não fica clara a identificação do ponto crítico $\mathrm{C}$ através do ensaio de sedimentação em proveta. Para contornar tal limitação, utilizam-se procedimentos auxiliares para a identificação do ponto crítico $\mathrm{C}$, como, por exemplo, o traçado das bissetrizes (Figura 3). Valadão (2007) reforça a importância da identificação dos limites entre as seções da curva de sedimentação nos métodos usuais de dimensionamento de espessadores que utilizam ensaios em provetas.

Analisando as equações utilizadas pelos métodos de dimensionamento (Tabela 1), verifica-se que o balanço de massa leva em consideração a total remoção de sólidos no clarificado, operação esta considerada como ideal. $\mathrm{Na}$ operação industrial, não é raro encontrar sólidos no overflow, sendo esta uma condição real.

Um fator de escala $(f)$ é, usualmente, utilizado para corrigir o diâmetro de espessadores, o qual é calculado através de ensaios de laboratório. Para esse fator, a literatura indica valores na faixa de $1,29<f<1,33$, para espessadores convencionais (Chaves, 1996) e $f=1,4$, para espessadores de lamelas (Kelly; Spottiswood, 1982). A utilização de um fator de escala no dimensionamento é justificada pelo fato de que os ensaios de sedimentação foram realizados em bateladas (sedimentação em provetas), enquanto que os equipamentos industriais vão operar em regime contínuo (Chaves, 1996). Gradientes de tempera- 
Thiago César de Souza Pinto et al.

Tabela 1 - Equacionamento dos métodos aplicados.

\begin{tabular}{c|c|c}
\hline EQUAÇÕES & Área & Variáveis \\
\hline $\begin{array}{c}\text { MéTODOS } \\
\text { Sólidos }\end{array}$ & $A=\frac{Q_{0} C_{0}}{F_{L}}$ & $F_{i}=C_{i} V_{i} ; \quad V_{i}=\frac{\Delta Z_{i}}{\Delta \theta_{i}}$ \\
\hline Talmadge-Fitch & $A=\frac{Q_{0} \cdot \theta_{E}}{Z o}$ & $Z_{E}=\frac{C_{0} \cdot Z_{0}}{C_{e}}$ \\
\hline Roberts & $Q a \cdot C a \cdot\left(\frac{1}{C c}-\frac{1}{C e}\right)$ \\
& $A=\frac{C c=\frac{Z_{0} \cdot C_{0}}{Z_{i c}} ; \quad u=\frac{Z_{i c}-Z_{c}}{\theta_{c}}}{}$ \\
\hline
\end{tabular}

Onde:

$F_{i}=$ fluxo de sólidos $\left(\mathrm{kg} / \mathrm{m}^{2} \mathrm{~h}\right)$.

$F_{L}=$ fluxo de sólidos limitante $\left(\mathrm{kg} / \mathrm{m}^{2} \mathrm{~h}\right)$.

$C_{i}=$ concentração de sólidos na alimentação do espessador $\left(\mathrm{kg} / \mathrm{m}^{3}\right)$.

$V i=$ velocidade inicial de sedimentação das partículas $(\mathrm{m} / \mathrm{h})$.

Qa ou $Q_{0}=$ vazão volumétrica de alimentação $\left(\mathrm{m}^{3} / \mathrm{h}\right)$.

Ca ou $C_{0}=$ concentração de sólidos na alimentação $\left(\mathrm{kg} / \mathrm{m}^{3}\right)$.

$\mathrm{Ce}=$ concentração de sólidos no underflow $\left(\mathrm{kg} / \mathrm{m}^{3}\right)$.

$C c=$ concentração de sólidos na fase inicial de compressão - concentração crítica $\left(\mathrm{kg} / \mathrm{m}^{3}\right)$.

$u=$ velocidade de sedimentação das partículas $(\mathrm{m} / \mathrm{h})$.

$\theta_{E}=$ tempo para se alcançar a concentração de sólidos desejada no underflow, $\mathrm{h}$.

$\theta_{c}=$ tempo para se alcançar a concentração de sólidos do underflow no ponto crítico, $\mathrm{h}$.

$A$ = área mínima do tanque espessador $\left(\mathrm{m}^{2}\right)$.

$Z$ = Altura da linha divisória entre o líquido clarificado e a polpa sob sedimentação.

$Z_{0}=$ altura inicial da proveta, $\mathrm{m}$.

$Z_{C}=$ altura dos sólidos na zona crítica, $m$.

$Z_{i c}=$ altura obtida graficamente, através da reta tangente que passa por $Z_{C}$ e $\theta_{C}, \mathrm{~m}$.

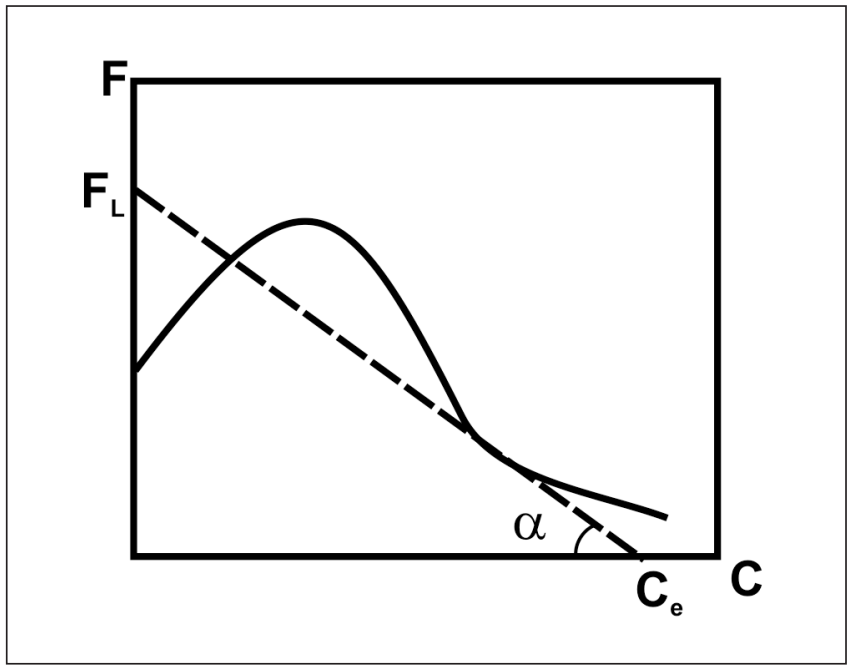

Figura 1 - Ilustração da identificação do fluxo de sólidos limitante $\left(F_{L}\right)$ na curva fluxo de sólidos em função da concentração (TFS).

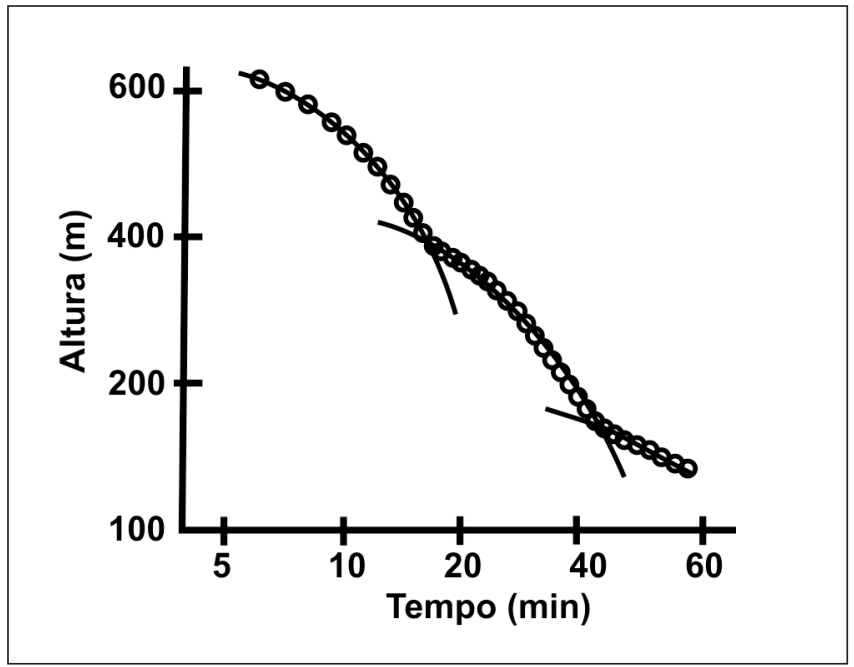

Figura 2 - Descontinuidades da curva altura da interface em função do tempo em papel log-log (Roberts). 


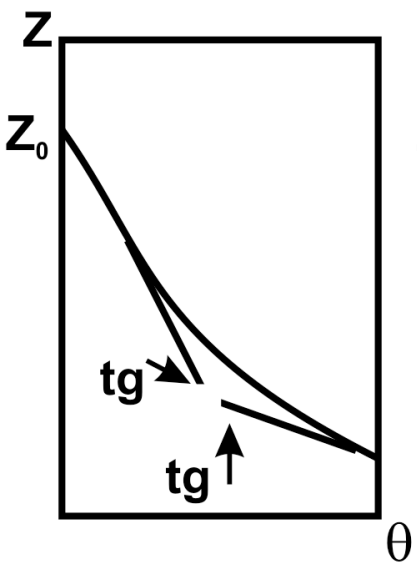

(a)

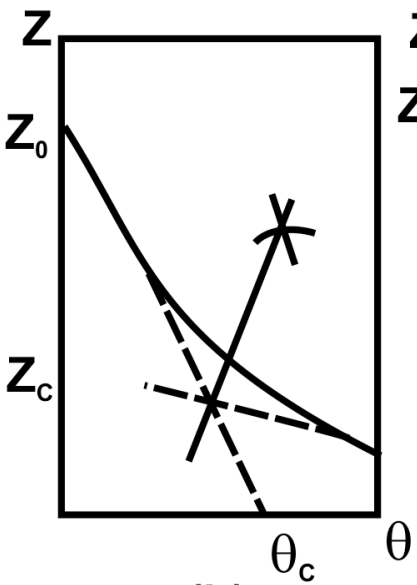

(b)

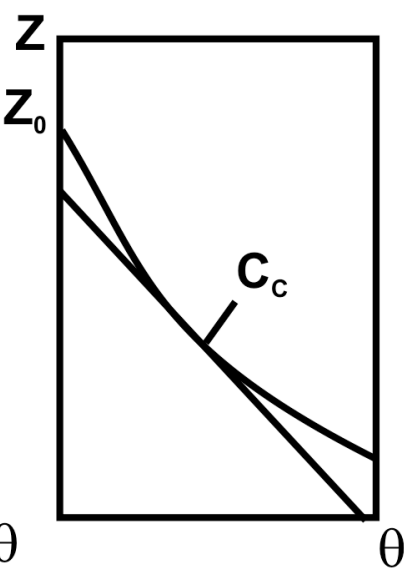

(c)

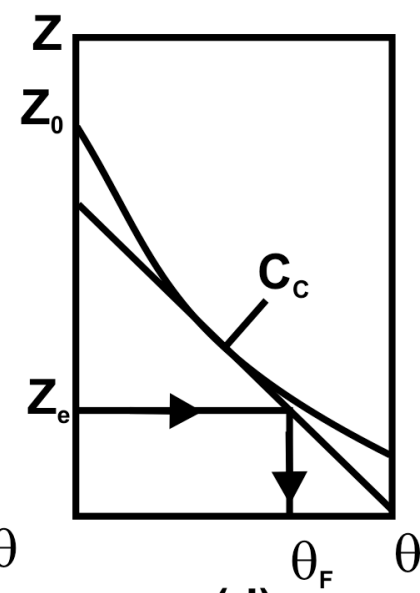

(d)

Figura 3 - Procedimento gráfico segundo Talmadge-Fitch (1955), apud Moraes (1990).

tura e possível formação de bolhas na polpa, oriundas de canais preferenciais de sedimentação, também justificam o uso do fator de escala (Moraes, 1990). Também é usual que engenheiros projetistas utilizem um fator de segurança $\left(f_{\mathrm{s}}\right)$, para que o equipamento, quando em operação, absorva variações operacionais. Isto se justifica pelo fato de os espessadores serem equipamentos de alto custo e, também, inflexíveis na sua operação (Chaves, 1996).

Em algumas operações industriais, utilizam-se reagentes auxiliares de desaguamento (coagulantes e/ou floculantes) com o objetivo de produzir agregados (flocos e/ou coágulos) que diminuam o tempo de sedimentação de tais opoerações. Uma vez que sinal e magnitude do potencial zeta $(\Psi z)$ das partículas influenciam nas forças de atração ou repulsão que atuam sobre as mesmas, podese controlar o estado de agregação da suspensão através da magnitude e do sinal de $\Psi z$ (Parfitt, 1969).

\section{Materiais e métodos}

\subsection{Materiais}

Utilizou-se salmoura oriunda de uma indústria localizada no Pólo Industrial de Cubatão-SP. Ela contém 313,9 g/1 de sólidos em suspensão e é submetida a um processo de clarificação através do uso de um espessador de $20 \mathrm{~m}$ de diâmetro. A caracterização física e química da salmoura é apresentada na Tabela 2, enquanto que, na Tabela 3, são apresentadas as informações sobre os sólidos suspensos na referida salmoura.

Tabela 2 - Caracterização da Fase Líquida.

\begin{tabular}{|c|c|c|c|}
\hline $\begin{array}{c}\text { Composição } \\
\text { Química }\end{array}$ & $\mathrm{pH}$ & $\begin{array}{c}\text { Viscosidade } \\
\left(25^{\circ} \mathrm{C}\right)\end{array}$ & $\begin{array}{c}\text { Massa } \\
\text { Específica }\end{array}$ \\
\hline$\% \mathrm{H}_{2} \mathrm{O}=73,46 \%$ & \multirow{6}{*}{11,2} & \multirow{6}{*}{ 2,05 mPa.s } & \multirow{6}{*}{$1.201,96 \mathrm{~kg} / \mathrm{m}^{3}$} \\
\hline$\% \mathrm{NaCl}=26,30 \%$ & & & \\
\hline $\mathrm{Na}_{2} \mathrm{CO}_{3}=0,09 \%$ & & & \\
\hline$\% \mathrm{CaCO}_{3}=0,07$ & & & \\
\hline $\mathrm{NaOH}=0,05 \%$ & & & \\
\hline$\% \mathrm{Mg}(\mathrm{OH})_{2}=0,03 \%$ & & & \\
\hline
\end{tabular}

Tabela 3 - Caracterização da Fase Sólida.

\begin{tabular}{c|c|c}
\hline $\begin{array}{c}\text { Composição } \\
\text { Química }\end{array}$ & $\begin{array}{c}\text { Potencial Zeta das } \\
\text { partículas ( }\end{array}$ & $\begin{array}{c}\text { Massa } \\
\text { Específica }\end{array}$ \\
\cline { 1 - 1 }$\% \mathrm{NaCl}=85,36 \%$ & & \\
\cline { 1 - 1 } $\mathrm{CaCO}_{3}=10,94 \%$ & $-19,0 \mathrm{mV}$ & $2.236,0 \mathrm{~kg} / \mathrm{m}^{3}$ \\
\cline { 1 - 1 } $\mathrm{Mg}(\mathrm{OH})_{2}=3,27 \%$ & & \\
\hline $\mathrm{Na}_{2} \mathrm{CO}_{3}=0,43 \%$ & & \\
\hline$\left.{ }^{*}\right)$ Suspensas na salmoura, em $\mathrm{pH}=11,2$. &
\end{tabular}




\subsection{Métodos}

Ensaios de sedimentação foram realizados em provetas de $2000 \mathrm{ml}$ obedecendo aos procedimentos padronizados pelo método do Fluxo de Sólidos, reportado por Metcalf et al. (1979), Talmadge et al. (1955) e Roberts (1949).

Durante a execução de cada ensaio de sedimentação, monitorou-se a altura (Z) da interface sólido-líquido (nas provetas graduadas), em função do tempo $(\theta)$, obtendo-se o comportamento de Z, em função de $\theta$. A concentração de sólidos (C) na alimentação, no underflow e no overflow do espessador foi obtida por secagem em estufa de volumes de polpa de $100 \mathrm{ml}$ até massa constante.

A massa específica (d) foi determinada através de picnômetro de $50 \mathrm{ml}$, coletando-se amostras da alimentação, do underflow e do overflow.

Medidas de potencial zeta das partículas submetidas à sedimentação foram realizadas no equipamento Zetasizer 3000, fabricado pela Malvern, usando-se a técnica da mobilidade eletroforética (convertida em potencial zeta através da equação de Smoluchowsk). A viscosidade da salmoura foi determinada em viscosímetro Brookfield modelo LVDV-E a $25^{\circ} \mathrm{C}$. Tratando-se a salmoura de um fluido newtoniano, a viscosidade foi calculada através da inclinação da reta tensão de cisalhamento $(\tau)$ versus taxa de deformação $(\Omega)$.

O espessador industrial que processa a salmoura estudada nesse trabalho exibe características que são apresentadas na Tabela 4, destacando-se o diâmetro de 20 m.

\section{Resultados e discussão}

Os resultados apresentados nas Tabelas 2 e 3 informam que os sólidos estão suspensos em uma salmoura que apresenta $\mathrm{pH}=11,2$, viscosidade de $2,05 \mathrm{mPa} . \mathrm{s}\left(\mathrm{a} 25^{\circ} \mathrm{C}\right)$ e massa específica de $1.026 \mathrm{~kg} / \mathrm{m}^{3}$. A magnitude da viscosidade se mostra muito superior à da água na mesma temperatura $(0,89 \mathrm{mPa} . \mathrm{s})$, todavia sua massa específica é somente 2,6\% superior à da água.

Analisando-se as informações apresentadas na Tabela 4, verifica-se que o espessador industrial é capaz de reduzir a concentração inicial de sólidos de $313,9 \mathrm{~g} / 1$ (na alimentação) para 141,5g/1 (no overflow). Por outro lado, o produto espessado (underflow) apresenta concentração de sólidos de 784,1g/1. Verifica-se, através das informações supracitadas, que o overflow apresenta alta concentração de sólidos.

Com base no potencial zeta $(-19 \mathrm{mV})$ das partículas suspensas na salmoura, pode-se inferir que as mesmas não apresentam considerável acúmulo de carga negativa na interface salmoura/solução. Tal comportamento indica que a repulsão eletrostática entre as partículas não é suficiente para evitar a coagulação das mesmas em grande extensão, durante a sedimentação.

A Figura 4 apresenta o comportamento da altura (Z) do limite de separação entre a fase clarificada e a zona de sedimentação versus tempo $(\theta)$. O comportamento de $Z$ versus $\theta$ ilustrado por tal curva é corroborado pela literatura corrente (Coe et alii, 1916 e Talmadge et alii, 1955).

Tabela 4 - Informações sobre espessador industrial cujo dimensionamento será validado.

\begin{tabular}{c|c}
\hline Parâmetros & Magnitude das variáveis \\
\hline Diâmetro do espessador & $20 \mathrm{~m}$ \\
\hline Fluxo de alimentação & Vazão $=80 \mathrm{~m}^{3} / \mathrm{h}$ \\
Fluxo no overflow & Massa específica $=1026,0 \mathrm{~kg} / \mathrm{m}^{3}$ \\
\hline Fluxo no underflow & Concentração de sólidos $=141,5 \mathrm{~g} / \mathrm{l}$ \\
& Massa específica $=1017,0 \mathrm{~kg} / \mathrm{m}^{3}$ \\
\hline
\end{tabular}

A Figura 5 apresenta o comportamento da sedimentação das partículas na salmoura através do procedimento gráfico utilizado por Roberts (1949) para dimensionamento de espessadores. A descontinuidade na curva indica o ponto crítico do espessamento, isto é, o início da zona de compressão. A Figura 6 ilustra o comportamento do fluxo de sólidos limitante $\left(\mathrm{F}_{\mathrm{L}}\right)$ versus concentração de sólidos na suspensão. Tal curva exibe forma bastante similar a outras curvas encontradas na literatura corrente (Chaves, 1996; Fitch, 1966 e Talmadge et alii, 1955).

Executando-se o dimensionamento do tanque espessador pelas três técnicas estudadas, obtiveram-se os resultados exibidos na Tabela 5, onde se observa que os valores calculados para área de sedimentação $\left(151,7-156,1 \mathrm{~m}^{2}\right)$ e, conseqüentemente, diâmetro do tanque (13,9 - 14,1m), exibiram valores muito próximos entre si, mostrando grande convergência entre os resultados das três técnicas.

Aplicando-se um fator de escala $(f=1,30)$ aos diâmetros determinados pelas três técnicas, obtiveram-se valores corrigidos para o diâmetro do espessador, sendo que tais valores estão apre- 


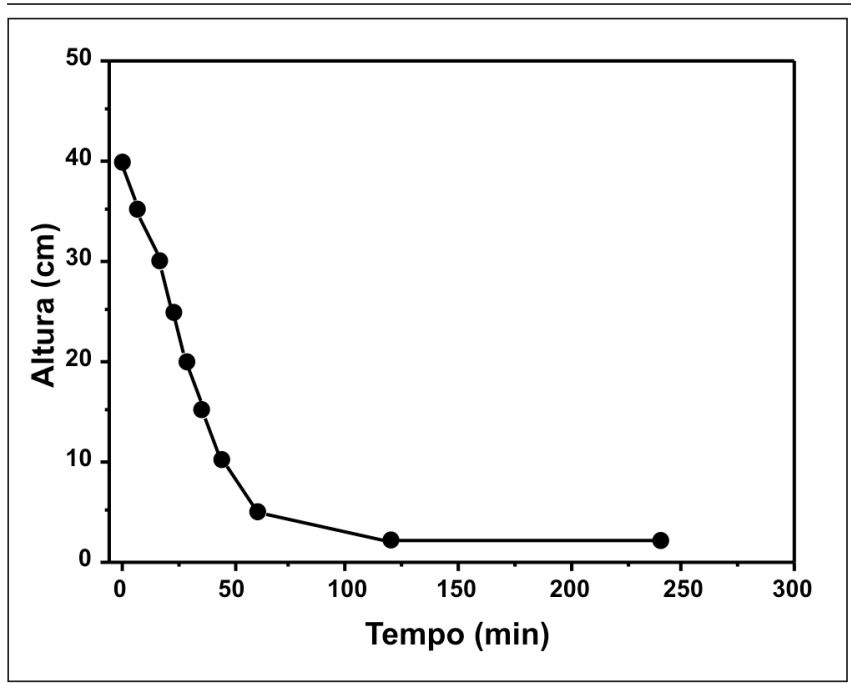

Figura 4 - Comportamento da altura em função do tempo do limite de separação entre fase clarificada e zona de sedimentação.

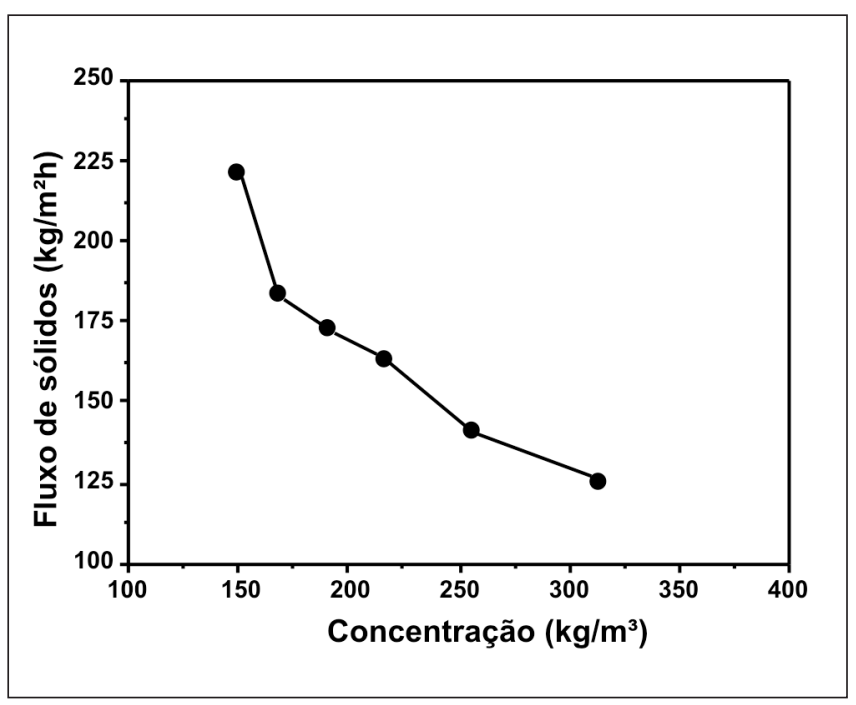

Figura 6 - Curva do Fluxo de Sólidos versus Concentração.

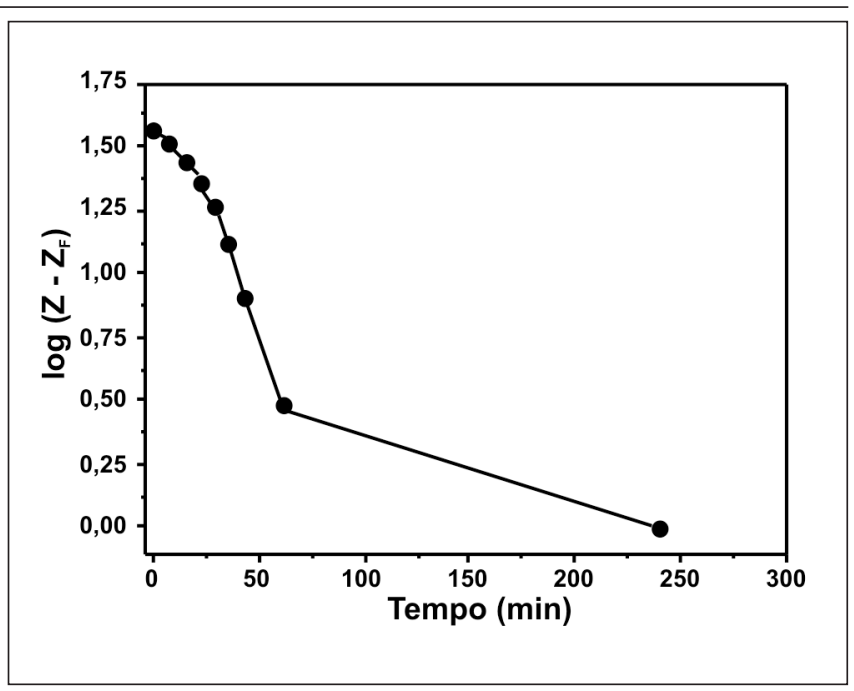

Figura 5 - Curva da técnica de Roberts aplicada à suspensão de salmoura.

sentados na Tabela 5. Comparando-se os diâmetros corrigidos (18,1-18,3m) com o diâmetro real (20m), verifica-se um desvio na faixa de $8,5-9,5 \%$. Tal discrepância poderia ser interpretada como um erro inerente às técnicas adotadas para dimensionamento (sedimentação em provetas). Tal interpretação poderia ser justificada em função da subjetividade dos métodos em identificar o início da zona de compressão ou, ainda, em função das dificuldades na leitura da interface sólido-líquido da polpa nos ensaios em provetas bem como nos procedimentos gráficos. Foi verificado, ainda, que o equipamento apresentou problemas na clarificação da salmoura, em função da alta taxa de sólidos no overflow. Problemas técnicos e operacionais podem ter contribuído para tal fato.

Tabela 5 - Dimensionamento do Espessador.

\begin{tabular}{c|c|c|c|c}
\hline \multirow{2}{*}{ Método } & \multicolumn{2}{|c|}{ Área $\left(m^{2}\right)$} & \multicolumn{2}{c}{ Diâmetro (m) } \\
\cline { 2 - 5 } & Calculada & Corrigida & Calculado & Corrigido \\
\hline TFS & 151,7 & 257,3 & 13,9 & 18,1 \\
\hline Talmadge-Fitch & 151,7 & 257,3 & 13,9 & 18,1 \\
\hline Roberts & 156,1 & 263,0 & 14,1 & 18.3 \\
\hline
\end{tabular}




\section{Conclusão}

Resultados de área $\left(\mathrm{A}=151,7-156,1 \mathrm{~m}^{2}\right)$ e diâmetro $(\mathrm{D}=13,9-14,1 \mathrm{~m})$ do espessador, calculados através das três técnicas usualmente citadas em literatura, convergiram para valores muito próximos, indicando coerência entre os procedimentos adotados através das referidas técnicas. Aplicando a esses valores um fator de escala $(f=1,3)$, foi possível encontrar D e A $\left(D=18,1\right.$ - 18,3m; $\left.A=257,3-263,0 \mathrm{~m}^{2}\right)$ com magnitude $10 \%$ inferior ao espessador real. Tais resultados validam as técnicas experimentais adotadas para o dimensionamento do espessador industrial dentro das condições estudadas.

\section{Referências bibliográficas}

CHAVES, A.P. et al. Espessamento. In: Teoria e prática no tratamento de minérios, Edição de Signus/Mineral.v. 2, cap.5, p.280-347, 1996.

COE, H.S., CLEVENGER, G.H. Methods for determining the capacities of slime settling tanks. In: Transactions of the American Institute of Mining, Metallurgical and Petroleum Engineers, v. 60, p. $356-358,1917$.
FITCH, B. A mechanism of sedimentation. In: Industrial and Engineering Chemistry, v.5, p. 129-134, 1966.

KELLY, E.G., SPOTTISWOOD, D.J. Introduction to mineral processing. Nova York: Wiley Interscience Inc., 1982. cap. 17, p. 327-342.

METCALF, EDDY, Inc. Wastewater engineering: treatment, disposal, reuse. (2 ed.). New York: McGraw-Hill, 1979. 920p.

MORAES JR., D. Separações sólido-líquido: moendas, centrífugas, espessadores e filtros. São Carlos: Ed. UFSCAR, 1990. 182 p.

PARFITT, D. G. Fundamental aspects of dispersion. In: PARFITT, D. G. (editor). Dispersion of solids in liquids: with special reference to pigments. Elsevier, Amsterdam, cap. 3, p. 81-121, 1969.

ROBERTS, E. J. Thickening, art or science. In: Mining Engineering, v.1, p. 61-64, 1949.

TALMADGE, W.P., FITCH, E.B. Determining thickener unit areas. In: Industrial and Engineering Chemistry, v.47, p.38-41, 1955.

VALADÃO, G.E.S., ARAÚJO, A.C. Espessamento. In: Introdução ao tratamento de minérios, Editora UFMG, cap.7, p. 142-152, 2007.

Artigo recebido em 23/06/2008 e aprovado em 14/04/2009.

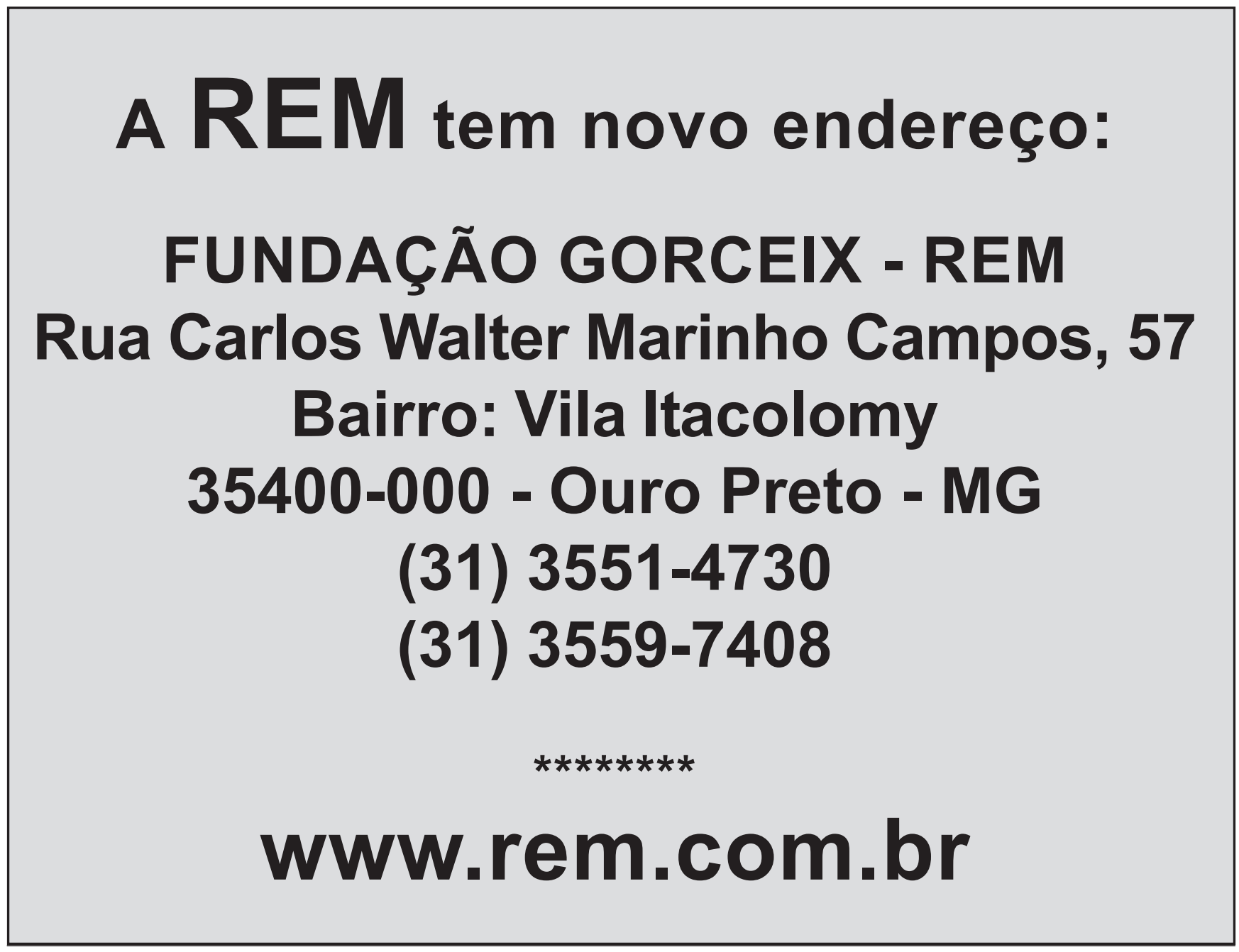

